\title{
DOPAMINERGIC GRAFTS IMPLANTED INTO THE NEONATAL OR ADULT STRIATUM: COMPARATIVE EFFECTS ON ROTATION AND PAW REACHING DEFICITS INDUCED BY SUBSEQUENT UNILATERAL NIGROSTRIATAL LESIONS IN ADULTHOOD
}

\author{
D. N. Abrous, ${ }^{*} \uparrow$ E. M. Torres $\ddagger$ and S. B. DunnetT $\ddagger$ \\ †INSERM U-259, Université de Bordeaux II, France \\ $\ddagger$ Department of Experimental Psychology, University of Cambridge, Cambridge, U.K.
}

\begin{abstract}
We have examined whether dopaminergic mesencephalic grafts implanted into neonates can provide more extensive protection against deficits induced by a subsequent unilateral lesion of the mesotelencephalic dopaminergic pathway than when the grafts are implanted in adulthood. A dopaminerich neuronal cell suspension obtained from embryonic day 14 mesencephali was injected unilaterally into the neostriatum of otherwise intact neonatal or adult rats at one day or two months of age, respectively. Two months later, the ipsilateral mesotelencephalic dopaminergic pathway was destroyed by unilateral injection of 6-hydroxydopamine. The behavioural effects of the grafts were evaluated in tests of drug-induced rotation and skilled paw reaching. After completion of the behavioural testing, animals were killed and brains were processed for tyrosine hydroxylase immunohistochemistry.

In rats receiving transplants as adults, grafts were compact and located in the neostriatum. In contrast, in rats receiving transplants neonatally, fewer dopaminergic neurons survived and they were dispersed over a large area of the host neostriatum and nucleus accumbens. After lesioning, all animals manifested strong rotation in response to amphetamine: this was not initially prevented by the grafts, made at either age, up to three months following the lesion, but was reduced in both groups of grafted rats by seven months after lesioning. This prolonged period for the development of recovery contrasts markedly with the rapid recovery obtained when similar grafts are implanted into the denervated neostriatum of adult rats that had received a prior 6-hydroxydopamine lesion. The development of apomorphine rotation, thought to reflect the development of receptor supersensitivity following lesions, was partially blocked to a similar extent by the grafts in both age groups. In contrast to their effects in the rotation tests, the dopaminergic grafts had no detectable effect on the profound contralateral deficit induced by the lesions in the paw-reaching test, whether implanted into neonatal or adult brains.

Thus, whereas the age of the host at the time of implantation can markedly influence the gross morphological organization of dopaminergic grafts implanted into the neostriatum, the functional effects were similar, whether the grafts were implanted into neonatal or adult hosts.
\end{abstract}

It is now well established that embryonic dopaminergic (DA) neurons implanted in the central nervous system of adult rats can exert a functional influence on the host. Never the less, when the functional capacities of grafted animals are studied in detail, the recovery brought about by these transplants is found to be incomplete. ${ }^{7,9,20,21,24-31,38-41,43,49,56}$

In attempting to identify the principles that govern the extent and limitations of recovery, one line of recent research has focused attention on the functional effect of DA grafts implanted into the neonatal brain. In an important study, Schwartz and Freed ${ }^{60}$ found that transplantation of DA neurons into newborn rats can protect at least some animals from the

*To whom correspondence should be addressed.

Abbreviations: BSA, bovine serum albumin; DA, dopaminergic; DNAse, deoxyribonuclease; 6-OHDA, 6-hydroxydopamine; PBS, phosphate-buffered saline; S-MEMHEPES, synthetic minimum essential medium-N-2hydroxyethylpiperazine- $N^{\prime}$-ethane sulphonic acid; TH, tyrosine hydroxylase. profound aphagia and adipsia induced by bilateral forebrain DA lesions made subsequently when the animals had grown to maturity. Since such regulatory deficits have been resistant to recovery when DA grafts are implanted into adult animals, ${ }^{25,26}$ they speculated that the most extensive recovery obtained when the grafts are implanted into neonates may be due to a greater capacity of the grafted embryonic neurons to become integrated anatomically into a developing (as opposed to a mature) host neural circuitry. ${ }^{60}$ Indeed, a facilitatory effect of immature brain over the anatomical incorporation of transplanted neurons has been described in a variety of different neuronal graft models. ${ }^{15-19,35-37,45-47,52,64,65,69}$ We have replicated the observation of Schwartz and Freed ${ }^{60}$ that neonatal implants can indeed protect against the regulatory deficits induced by subsequent bilateral 6-hydroxydopamine (6-OHDA) lesions in at least some animals, and found that the location of the grafts in the caudal ventral striatum was an important factor in their efficacy. ${ }^{58,59}$ 
However, in previous work, implantation of embryonic DA neurons into the striatal complex of newborn rats in which the ascending dopaminergic pathways had been previously destroyed did not exhibit any greater functional improvement than that observed in adults. For example, Abrous and colleagues found that nigral grafts were no more effective at alleviating deficits in hoarding and schedule-induced polidipsia following transplantation into neonatally lesioned rats than they were when the lesions and grafts were made in adulthood. ${ }^{2}$ However, the studies differ in several important respects, which may account for the different outcomes. Firstly, they are based on tests of different components of the nigrostriatal denervation syndrome: basic regulatory deficits involving aphagia, adipsia and hyperkinesia on the one hand, and tests of possibly more complex functions including hoarding, schedule-induced polidipsia and spatial orientation, on the other. Secondly, the two sets of studies differ importantly in the timing of the lesions and graft implantation. On the one hand, the grafts were implanted into the brains of otherwise intact pups and the functional effects studied prophylactically on the consequences of lesions made once the rats had reached adulthood. ${ }^{58.59}$ In the other study, ${ }^{2}$ lesions were made in newborn pups, several days prior to transplantation, following the conventional sequence of operations used in studies of the functional effects of DA grafts implanted in adult animals. Thirdly, both previous studies have evaluated the consequences of neonatal grafts on behaviours associated with bilateral lesions, whether made in neonatal or adult animals, whereas the great majority of studies on the functional effects of grafts in adult animals have been based on the unilateral lesion model. ${ }^{8,9,24,27,30,31,38,44,49}$ Indeed, other studies have suggested that DA grafts in adult brain can exert a prophylactic effect on the drug-induced rotation following a subsequent nigrostriatal lesion. ${ }^{33}$

In the present study we have evaluated the relative functional incorporation of DA grafts into the neonatal and adult brain by comparing the functional efficacy of DA neurons implanted into the neonatal or adult neostriatum in alleviating deficits associated with a subsequent unilateral nigrostriatal destruction in adulthood. In particular, the prophylactic effects of the grafts have been investigated with drug-induced turning behaviours, which are particularly sensitive for assessing nigrostriatal lesions and DA-rich grafts, ${ }^{7-9,27,38,44}$ and with a skilled paw reaching task which is sensitive to nigrostriatal damage ${ }^{61,68}$ and which has been shown to be resistant to alleviation by DA grafts in adult rats. ${ }^{5.31 .54}$

\section{EXPERIMENTAL PROCEDURES}

\section{Subjects}

A total of 51 female Sprague-Dawley rats (Olac, Bicester, Oxon, U.K.) were used as experimental animals; 24 received operations at one day of age and the remaining 27 received the first experimental treatment at two months of age (see Table 1). For the neonatal procedures, litters were cullcd to 10 within $24 \mathrm{~h}$ of birth and reared by the mothers until weaning at 21 days of age. Following weaning, all animals were housed in groups of five with ad libitum access to food and water, and under a natural light/dark cycle. Graft tissues were dissected from E14 embryos obtained by Caesarian section of pregnant rats of the same outbred strain.

Six counterbalanced groups were employed: two graft groups received DA-rich grafts at either one day or two months of age, followed by lesions two months later; two parallel lesion groups received sham grafts but similar lesions, at two and four months of age; and two parallel control groups received both sham grafts and sham lesions. Data from an additional group receiving a nigrostriatal lesion in adulthood three weeks prior to the graft surgery (which was part of another experiment, but was prepared from the same graft suspension) are included for comparison with the conventional sequence of operations.

\section{Transplantation}

Grafting was performed according to a previously described method. ${ }^{40}$ The ventral mesencephalon was dissected from 35 embryos (three pregnant mothers) and collected in cold phosphate-buffered saline (PBS, pH 7.2) supplemented with $10 \mathrm{mM}$ glucose. Pooled tissue pieces were submitted to a brief $(5 \mathrm{~min}$ ) enzymatic digestion with $1 \%$ trypsin (Sigma grade II crude) in PBS with $0.1 \%$ DNAse added (Sigma). After washing with PBS, a synthetic culture medium was added (S-MEM-HEPES, Gibco-BRL, supplemented with $2 \mathrm{mM}$ glutamine, $14 \mathrm{mM}$ glucose, $0.02 \%$ trypsin inhibitor and $0.1 \%$ DNAse, Sigma) and a cellular suspension was obtained by repeated aspiration through a small-bore Pasteur pipette. Following a brief centrifugation $(1000 \mathrm{~g}$, $10 \mathrm{~min}$ ) to remove the supernatant, cells were resuspended

Table 1. Experimental groups

\begin{tabular}{|c|c|c|c|c|c|c|}
\hline \multirow[b]{2}{*}{ Group } & \multirow[b]{2}{*}{ Name } & \multirow[b]{2}{*}{$n$} & \multicolumn{2}{|c|}{ Age at grafting } & \multicolumn{2}{|c|}{ Age at lesion (w.r.t. graft) } \\
\hline & & & 1 day & 2 months & 3 weeks before & 2 months after \\
\hline Control rats & (SH) & $(10)$ & & & & \\
\hline Neonatal sham & NS & 5 & sham & -- & - & sham \\
\hline Adult sham & AS & 5 & - & sham & - & sham \\
\hline Lesion rats & (LE) & (14) & & & & \\
\hline Neonatal lesion & NL & 8 & sham & - & - & lesion \\
\hline Adult lesion & $\mathrm{AL}$ & 6 & - & sham & - & lesion \\
\hline Neonatal graft & NG & I1 & graft & - & - & lesion \\
\hline Adult graft & $\mathrm{AG}$ & 10 & - & graft & - & lesion \\
\hline $\begin{array}{l}\text { Lesion then } \\
\text { adult graft }\end{array}$ & LAG & 6 & - & graft & lesion & — \\
\hline
\end{tabular}


in S-MEM-HEPES containing $2 \mathrm{mM}$ glutamine, $14 \mathrm{mM}$ glucose and $0.2 \%$ bovine serum albumin (BSA, Sigma). The final suspension was kept on ice in glass tubes and was used for up to $4 \mathrm{~h}$ after preparation. The number of injected viable cells was approximately 130,000 cells/ $\mu 1$, as evaluated by dye exclusion. A 3- $\mu$ l aliquot of this suspension was injected stereotaxically into the host neostriatum via a glass cannula ( $100 \mu \mathrm{m}$ external diameter) attached directly onto a $10 \mu 1$ glass syringe.

Rats were anaesthetized either with ether (neonates) or $3 \mathrm{ml} / \mathrm{kg}$ equithesin i.p. (adults) and placed in a stereotaxic frame. The neonatal rats received an injection into the left neostriatum at the following stereotaxic coordinates: L $1.5 \mathrm{~mm}, \mathrm{AP}+1.5 \mathrm{~mm}$ from bregma, $\mathrm{V}-3.5 \mathrm{~mm}$ below the level of the skull, with the incisor bar at the level of the interaural line. The adult rats each received an injection at the following coordinates: $\mathrm{L} 2.5 \mathrm{~mm}, \mathrm{AP}+2.5 \mathrm{~mm}$, $\mathrm{V}-5.5 \mathrm{~mm}$, with the incisor bar set $5 \mathrm{~mm}$ above the level of the interaural line. The injections were delivered slowly over $3 \mathrm{~min}$ with a further $3 \mathrm{~min}$ allowed for diffusion prior to cannula retraction. All lesion and control groups received sham grafts involving an identical injection of $3 \mu$ l S-MEM-HEPES containing $0.2 \%$ BSA at either neonatal or two months of age (see Table 1). An antibiotic powder was applied externally, the skin sutured, the pups returned to their mothers and the adult rats to their home cage.

\section{Lesions}

The mesotelencephalic system of grafted rats was lesioned by the stereotaxic injection of 6-OHDA hydrobromide (Sigma) unilaterally into the left medial forebrain bundle of adult rats under equithesin anaesthesia $(3 \mathrm{ml} / \mathrm{kg})$. The neurotoxin was dissolved at a concentration of $4 \mu \mathrm{g}$ (free base weight $) / \mu 10.9 \%$ saline $-0.01 \%$ ascorbic acid. A total volume of $2 \times 1.5 \mu 1$ was injected stereotaxically over $4 \mathrm{~min}$ via a 30 -gauge stainless steel cannula at $\mathrm{L} 1.6 \mathrm{~mm}$, AP 0 and $-1 \mathrm{~mm}$ relative to bregma, $V-8.6 \mathrm{~mm}$ from the level of the skull, with the incisor bar set $5 \mathrm{~mm}$ above the interaural line. Following completion of the injection, the cannula was left in place for an additional 2 min to allow for diffusion of the neurotoxin away from the injection site.

The lesion was performed two months after the grafting procedure in the neonatal lesion, neonatal graft, adult lesion and adult graft groups, and three weeks prior to the grafts, at six weeks of age in the lesion then adult graft group (see Table 1). The two control groups received sham lesions by identical injection of ascorbate-saline solution alone.

\section{Behavioural testing}

Rotation. Animals were tested in automated rotometer bowls ${ }^{66}$ and the number of full $360^{\circ}$ turns per minute was recorded automatically by an on-line connection to a microcomputer. Rotations in the ipsilateral and contralateral directions were counted separately, and the analyses were based on the net (ipsilateral minus contralateral) scores.

For the six counterbalanced groups, rotation was tested at one week, one, two, three and seven months after the lesion over $300 \mathrm{~min}$ following i.p. injection of $5 \mathrm{mg} / \mathrm{kg}$ D-amphetamine sulphate (Sigma), and approximately one week later over $60 \mathrm{~min}$ following s.c. injection of $0.1 \mathrm{mg} / \mathrm{kg}$ apomorphine hydrochloride (Sigma). Amphetamine-induced rotation was examined in the lesion then adult graft rats two weeks following the lesion and again one, two, three and seven months after grafting. For the last time point (seven months), the amphetamine-induced rotation task was carried out two days after the completion of the skilled paw reaching task, followed one week later by apomorphineinduced rotation.

Skilled pow reaching: test apparatus. Paw reaching was measured using a modified version of the "staircase" test apparatus described and illustrated by Montoya and colleagues $^{54.55}$ The apparatus consists of a clear Perspex chamber $(203 \mathrm{~mm}$ long $\times 108 \mathrm{~mm}$ high $\times 103 \mathrm{~mm}$ wide) with a hinged lid, into which the rat is placed. Leading off this is a narrower compartment $(165 \mathrm{~mm}$ long $\times 108 \mathrm{~mm}$ high $\times 60 \mathrm{~mm}$ wide) with a central raised platform running along its length, creating a $19-\mathrm{mm}$-wide trough on either side. The narrowness of the side compartment prevents rats from turning rounds, so that the rat can only use its left paw for reaching into the left trough and its right paw for reaching into the right trough. The top surface of the platform is $35 \mathrm{~mm}$ wide and overhangs the sides so that the rats cannot simply scrape food pellets up the side of the platform. A removable double staircase is inserted into the end of the box, sliding into the troughs to either side of the central platform. Each of the eight steps of the staircase contains a small 3-mm-deep well and two 45 -mg chow pellets (Custom Biological Inc.) are placed in each well. The highest step of the staircase is $13 \mathrm{~mm}$ below the central platform and the bottom step is $64 \mathrm{~mm}$ below the platform. A hungry animal can collect pellets by reaching down into the trough: the number of steps from which pellets are displaced provides an index of how far the rat can reach and the number of pellets remaining at the end of the test indicates the rat's success in grasping and retrieving the pellets.

Procedure. Paw reaching was studied three months after the lesion, five months after grafting, in the six counterbalanced experimental groups. Animals were placed on a food-deprivation schedule, being fed 8-12 g of lab chow at the end of each afternoon to maintain them at $90 \%$ of their free-feeding weight. Two $45-\mathrm{mg}$ chow pellets were placed into each well of the double staircase of the test apparatus. Animals were placed into the test boxes for $10 \mathrm{~min}$. Then the staircase was removed and the number of remaining pellets was counted. Training was conducted in three stages:

(i) During the first stage, both sides were baited. Animals received 31 daily tests over one month. This stage allowed the rats' reaching ability with the two paws to be determined, from which was determined the paw preference of each animal: the "preferred" paw was defined for each rat as the paw with which each rat obtained more pellets. This was the paw ipsilateral to the side of the lesion in all rats of both the lesion and the graft groups.

(ii) During the second phase, animals were trained to use the paw previously determined as the "non-preferred paw" (contralateral to the lesions in all rats of both the lesion and the graft groups). For this purpose, just that side of the staircase accessible by the animals using the non-preferred paw was baited. Animals received 31 daily tests over a second one month period.

(iii) During the third stage, the animals received five tests over five days with both staircases baited, as in stage (i), in order to evaluate the effect of forced paw reaching on their reaching preference.

\section{Immunohistochemistry}

Animals were killed for inmunohistochemical analysis 10 months after the lesions, after completion of all behavioural testing. Under general barbiturate anaesthesia, they were perfused transcardially with $50 \mathrm{ml}$ PBS (pH 7.2) containing $5 \times 10^{4} \mathrm{IU} / \mathrm{ml}$ heparin, followed by $300 \mathrm{ml}$ of $4 \%$ paraformaldehyde in $0.1 \mathrm{M}$ phosphate buffer containing $14 \%$ of saturated picric acid solution buffered to $\mathrm{pH} 7.4$. Following a $24 \mathrm{~h}$ postfixation in the same fixative, $50-\mu \mathrm{m}$-thick coronal sections were cut using a Vibratome (Oxford Instruments). Free-floating sections were processed through a standard immunohistuchemical procedure ${ }^{6}$ in order to stain for tyrosine hydroxylase (TH, used at 1:5000 dilution, Boy, Paris, France). TH-positive staining was visualized by the biotin-streptavidin technique (ABC kit, Dako Labs, Glostrup, Denmark) using 3,3'-diaminobenzidine as the chromogen. 


\section{Statistical analysis}

All data were analysed by an ANOVA and in cases where there was a significant interaction, groups were compared using a Newman-Keuls test. The survival of DA neurons implanted in neonatal or adult hosts has been compared using a Student's $i$-test

\section{RESULTS}

\section{Histochemistry}

Injection of 6-OHDA into the medial forebrain bundle caused an almost total loss of TH-positive neurons from both the substantia nigra and the ventral tegmental area. As illustrated in Fig. la, the ventral mesencephalon was similarly depleted with virtually total loss of all TH-positive cells from the substantia nigra and no more than $10 \%$ of cells remaining in the ventral tegmental area in all animals in both the graft and lesion groups. In lesioned animals, this was accompanied by a total loss of TH-immunoreactive terminals in the neostriatum and the nucleus accumbens ipsilateral to the injection side (Fig. 1b). Although terminal denervation due to the lesion was masked by the grafts in all transplant groups, it was assumed to be comparable based on the similar loss of cells in the ventral mesencephalon.

In rats grafted in adulthood (groups adult graft and lesion then adult graft), transplants formed a compact tissue mass with a drop-like shape localized in the central part of the neostriatum in all cases (Fig. 1c). All grafts contained numerous TH-positive neurons with large cell bodies and sprouting neurites. In addition, very small, round TH-positive cells with neurites were also observed within the grafts. The remainder of the bulk of each graft, comprising the great majority of all cells, was non-immunoreactive for TH. The number of large TH-positive cells within the grafts, believed to be neurons, ranged from 198 to 3023 (mean $=1599 \pm 304$, using the Abercrombie correction procedure).' These grafts gave rise to a rich TH-positive reinnervation which extended to nearly
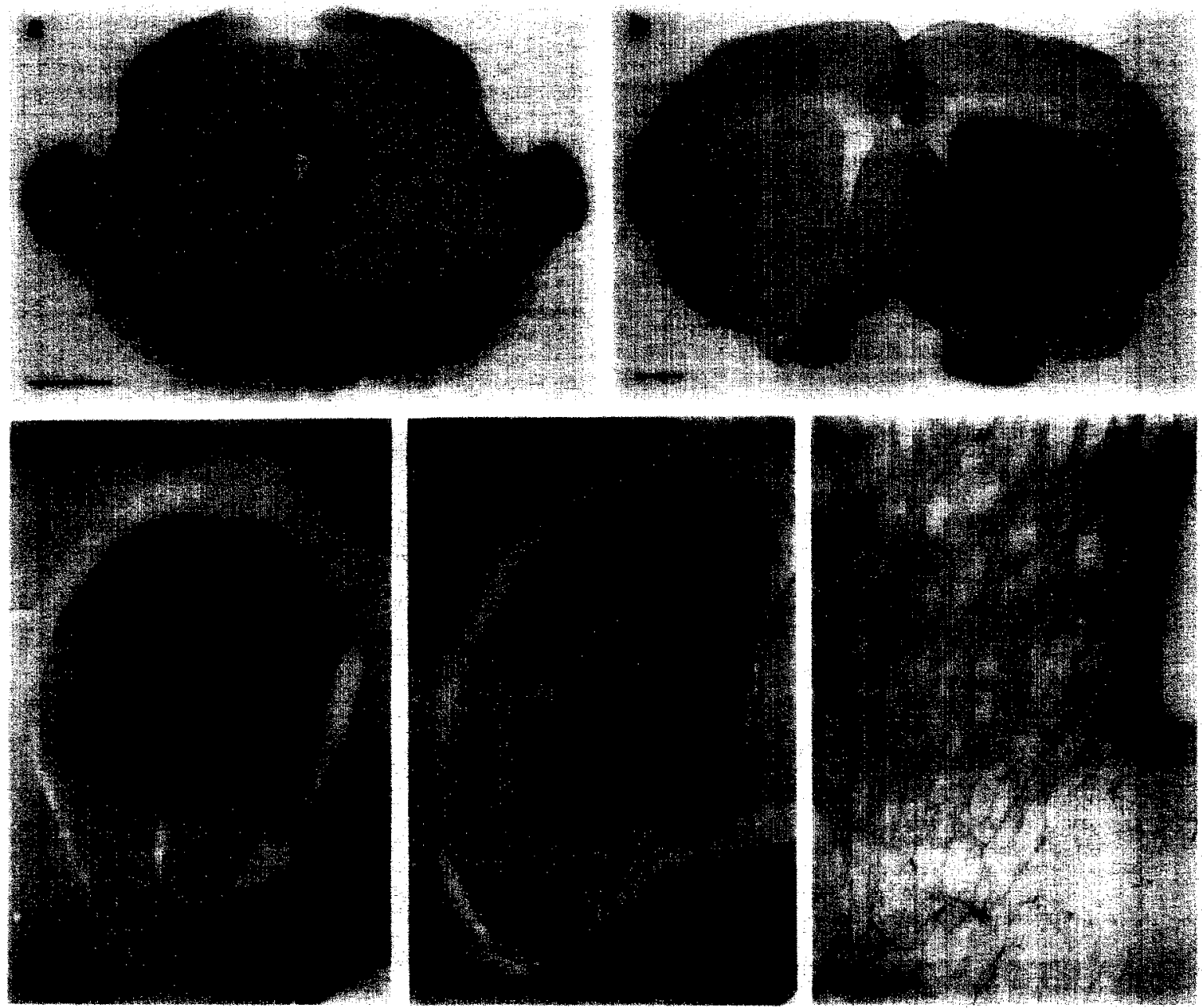

Fig. 1. Photomicrographs of TH immunohistochemistry on sections from lesioned and grafted animals. (a) Mesencephalon of a lesioned rat. (b) Striatal region of a lesioned rat. (c) Striatal region of an animal grafted at adulthood. (d) Striatal region of an animal grafted neonatally. (e) High magnification of a graft implanted in newborn rats. Seale bars $=100 \mu \mathrm{m}$. 
all the neostriatum. There were no detectable differences between the grafts implanted into the intact and the lesioned neostriatum (groups adult graft and lesion then adult graft, respectively).

In contrast, in most cases grafted neonatally (group neonatal graft), no distinct circumscribed mass of grafted tissue was apparent. Rather, TH-positive neurons were scattered throughout the striatal complex (Fig. 1d, e) and located either in the nucleus accumbens (two cases), in the medial or central parts of the neostriatum (five cases) or in both locations (four cases). In two cases, TH-positive neurons were also found in the contralateral intact neostriatum. The survival and location of non-dopaminergic (THnegative) cells injected in these grafts in neonates cannot be determined in the absence of explicit labelling of the graft cells. Small clumps of graft tissue were seen only when the neonatal grafts were localized in the dorsomedial corner of the neostriatum next to or merging into the lateral ventricle, and in these two cases the graft masses contained many THnegative as well as the stained TH-positive neurons. In neonatally grafted rats, all surviving TH-positive cells had large cell bodies giving rise to multiple neurites. The number of surviving TH-positive neurons $($ mean $=888 \pm 243 ;$ range: $233-2942$ ) was lower than that in adult grafted rats, which approached, but did not achieve, statistical significance ( $t=1.91$ with 19 d.f., $P=0.07$, based on a two-tailed hypothesis).

The location and density of fibre reinnervation of the host neostriatum was more patchy and scattered, depending on where the grafted cells had themselves become established within the host neuropil. For TH-positive neurons located in the nucleus accumbens, TH-positive fibres were found within this structure and within the ventral part (lateral and medial) of the neostriatum. TH-positive grafted neurons localized in the medial part of the neostriatum lining the ventricle reinnervated the body of the neostriatum, but this outgrowth generally did not reach the lateral margins of the nucleus. In other cases where the neurons were scattered throughout the central neostriatum, axon outgrowth extended to reinnervate the whole nucleus. In the two cases in which a distinct graft mass was seen to merge with the lateral ventricle, adjacent to the corpus callosum, a number of neurons extended neurites along the corpus callosum as well as into the neostriatum itself.

The differences in the anatomical appearance of DA grafts implanted into hosts of different ages closely matches those seen in previous studies. ${ }^{2,4,43,58}$

\section{Behavioural tests}

There was no difference on any test in the behavioural performances of the two sham groups (adult and neonatal) or of the two lesioned groups (adult and neonatal). Consequently, the behavioural results of these two pairs of groups have been pooled to form a combined sham group $(n=10)$ and a combined lesioned group $(n=14)$ respectively.

\section{Amphetamine-induced turning behaviour}

The effect of $5 \mathrm{mg} / \mathrm{kg} \mathrm{D}$-amphetamine on rotational activity measured at different times after surgery is illustrated in Fig. 2. Sham animals did not exhibit rotational activity after amphetamine administration. In contrast, amphetamine induced a strong rotation toward the lesioned side in lesioned animals, one week after lesioning. This ipsilateral bias increased in the period from one week to two months after lesioning and remained stable afterwards.

Animals lesioned and then grafted in adulthood exhibited an ipsilateral rotation before grafting which was compensated two months after grafting. Three months later, an overcompensation was observed and lesion then adult graft animals rotated contralaterally, i.e. away from the grafted side.

In animals grafted as adults, amphetamine induced a strong ipsilateral rotation during the three months following the subsequent lesion, which was similar to that observed in the lesioned group. Only by seven months after the lesion did the graft appear to be effective in decreasing this ipsilateral rotation. By this time, four animals had overcompensated (i.e. showed a net rotation score contralateral to the side of lesion and graft), four others had strongly reduced their initial ipsilateral rotation and two animals were still rotating ipsilaterally.

Animals grafted as neonates and subsequently lesioned and tested in adulthood exhibited an overall ipsilateral rotation which was initially weaker than that observed for the lesioned and adult graft groups. However, the rotational asymmetry increased so that by three months after the lesion, the neonatal graft group was turning to a similar extent as the lesioned and adult graft groups. By seven months after grafting, the neonatal graft group showed a similar degree

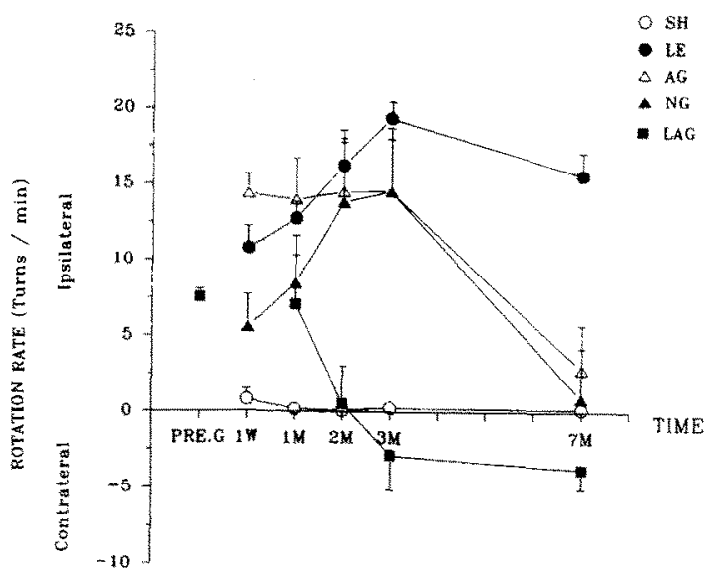

Fig. 2. Rotation activity induced by D-amphetamine $(5 \mathrm{mg} / \mathrm{kg})$. Results are expressed as mean rotation rate (turns/min, mean \pm S.E.M.) calculated over the first hour following the injection of the drug. Rotation was tested one week, one, two, three and seven months ( $1 \mathrm{~W}, 1 \mathrm{M}, 2 \mathrm{M}$, $3 \mathrm{M}$ and $7 \mathrm{M}$ respectively) after the lesion. For lesion then adult graft (LAG) rats, rotation was also examined before grafting (PRE.G). AG, adult graft; LE, lesioned; $N G$. neonatal graft; SH, sham. 
of compensation overall as seen in the adult graft group, of which eight animals out of 11 had compensated, and five animals exhibited a strong contralateral rotation.

Analysis of variance was used to compare the time course of recovery in the neonatal and adult graft groups compared to the lesion and sham groups. This indicated a very highly significant group $\times$ test interaction $\left(F_{12,148}=4.63, \quad P<0.001\right)$. Subsequent Newman-Keuls tests for multiple comparisons indicated that on the first four tests up to three months after the lesion, the lesion and the two adult and neonatal graft groups were not statistically different, whereas by seven months after the lesion, the two grafted groups rotated significantly less than lesioned rats. They did not significantly differ from each other or from the sham-operated control rats, whereas they were statistically different up to three nonths (control < lesion, adult graft, lesion then adult graft, $P<0.01$ at all time points; control $<$ neonatal graft: one week and three months, $P<0.05$, one and three months, $P<0.01$ ).

\section{Apomorphine-induced turning behaviour}

The effect of $0.1 \mathrm{mg} / \mathrm{kg}$ apomorphine on rotational activity measurcd at different times after surgery is illustrated in Fig. 3. Apomorphine had no effect on the rotational activity of sham animals. Two weeks after lesioning a strong contralateral rotation was observed in the lesioned group, which increased between one and two months and persisted over seven months of testing. Two weeks after the lesion, animals in the adult graft group rotated contralaterally but to a lesser extent than that observed for the lesioned group. In the period from two weeks to three months after the lesion, this contralateral rotation activity increased slightly but never reached the intensity of rotation observed in the lesioned group. Between three and seven months postlesion, there was a slight decrease of this contralateral rotation. The behaviour of animals grafted at the neonatal stage was similar

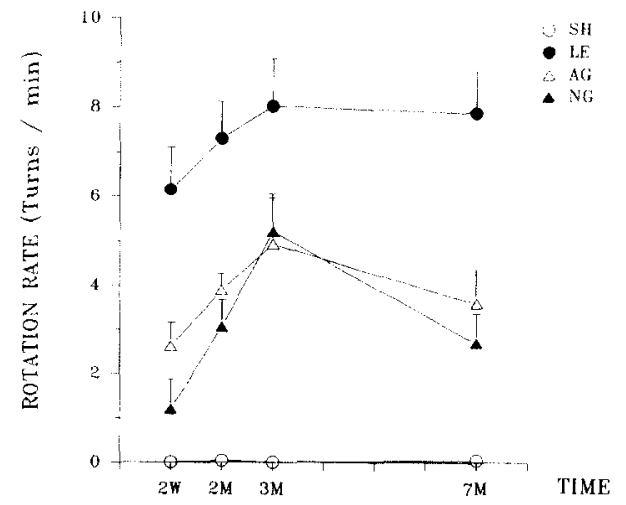

Fig. 3. Rotation activity induced by apomorphine $(0.1 \mathrm{mg} / \mathrm{kg})$. Results are expressed as mean rotation rate (turns/min, mean \pm S.E.M.) over 1 h. Rotation was examined two weeks, two, three and seven months $(2 \mathrm{~W}, 2 \mathrm{M}$, $3 \mathrm{M}$ and $7 \mathrm{M}$ respectively) after the lesion. to that observed for animals of the adult graft group. An overall analysis confirmed a main group effect $\left(F_{3,34}=14.55, P<0.001\right)$, as well as a significant group by session interaction $\left(F_{9,162}=1.98, P<0.05\right)$. Subsequent Newman-Keuls comparisons indicated the lesion group rotated at a higher rate than the graft groups at all time points (lesioned $>$ adult graft $=$ neonatal graft, all $P<0.01$ ). Animals of the lesion and the graft groups rotated more than control rats $(P<0.01)$ with the exception of the neonatal graft group, which did not statistically differ from the control group two weeks after the lesion.

\section{Skilled paw reaching}

Overall performance on the paw reaching task was assessed in terms of the total number of pellets collected by each group on each side of the apparatus during the three stages of the experiment, as shown in Fig. 4. During the first stage of the experiment (Fig. 4A), sham control animals learned to reach for food from either side of the central platform in the staircase boxes. They exhibited a slight lateralization. and the side on which more pellets were collected was defined as the "preferred" paw. This bias was dramatically enhanced by the lesion. Whereas the lesion rats showed no deficit in reaching with the paw ipsilateral to the lesion side, they manifested marked deficits in reaching using their contralateral paw. Thus, the "preferred" paw was the paw ipsilateral to the lesion in every case. The postlesion deficit was not influenced by DA grafts implanted either in adult or neonatal rats. Analysis of variance confirmed that animals in the different experimental groups were reaching for food with their preferred paw in a similar fashion (restricted main group effect: $F_{3.38}=0.54$; n.s.). By contrast, they were not using their nonpreferred paw to the same extent (restricted main group effect: $F_{3.38}=18.11, P<0.001 ;$ lesioned $=$ adult graft $=$ neonatal graft $>$ control; $P<0.01$ by Newman-Keuls test).

With further training, when forced to use their non-preferred paw (Fig. 4B), sham control animals manifested a further slight improvement in their reaching performance. In the lesion and graft groups, it was in cvery case the paw contralateral to the lesion which underwent additional training. Although the lesion and two graft groups also showed a modest improvement in use of the contralateral limb with further training, this never overcame the very marked impairments in comparison with the performance of the control rats or their own performance with the ipsilateral paw (see Fig 4). Analysis of variance again confirmed that these effects were highly significant (group effect: $F_{3,38}=29.33, \quad P<0.001$; lesioned $=$ adult graft $=$ neonatal graft $>$ control; $P<0.01$ ). Analysis of variance comparing the innprovement of performance with the "non-preferred" paw between the first and second stages of this experiment indicated no significant group by stage interaction $\left(F_{3.38}=0.84\right.$; n.s. $)$. 


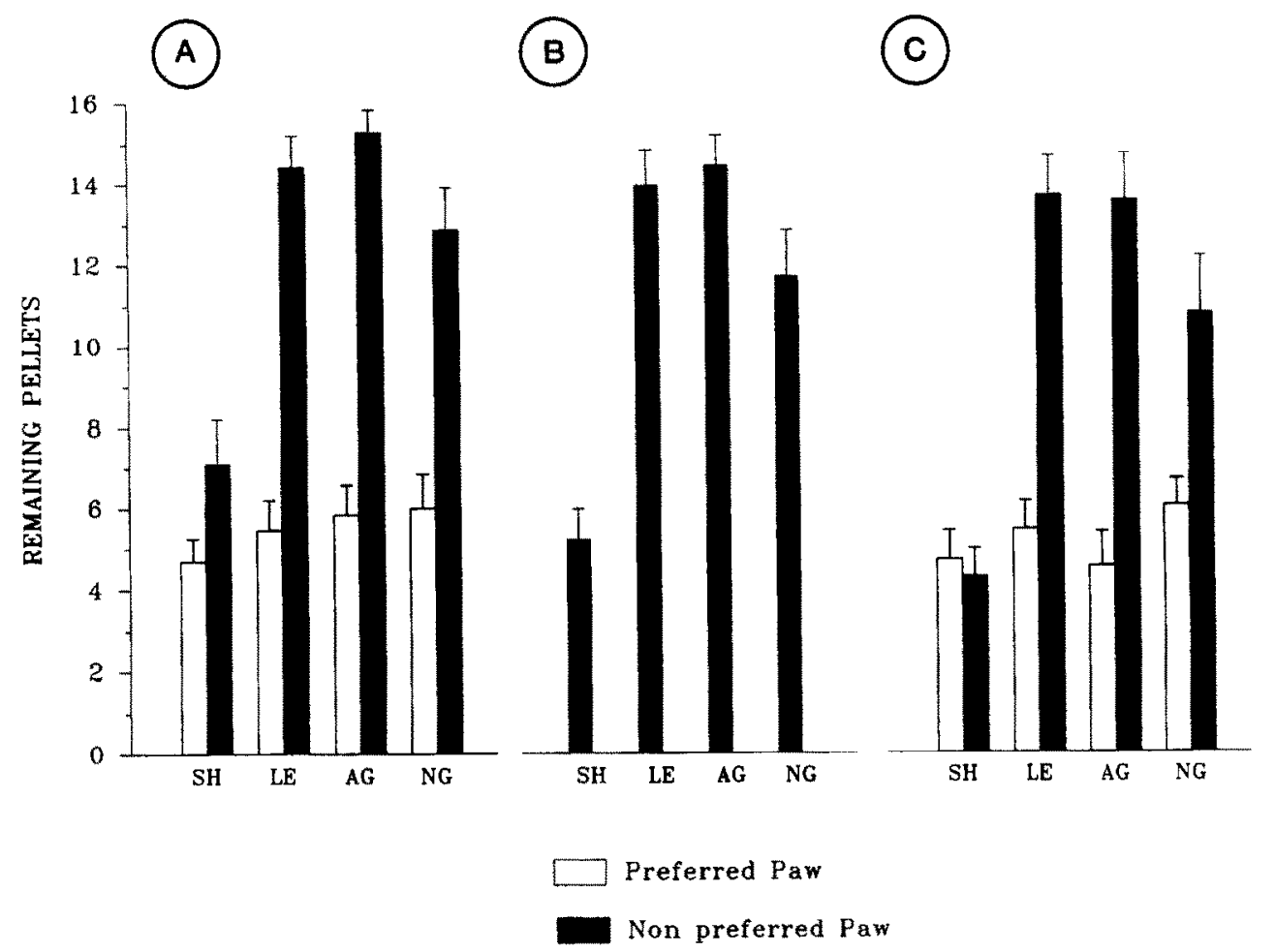

Fig. 4. Skilled paw reaching. (A) Spontaneous skilled paw reaching. (B) Forced paw use. (C) Spontaneous skilled paw reaching. Results are expressed as the average (mean \pm S.E.M.) number of pellets remaining in the staircase test over the last five trials.

When retested in standard conditions (Fig. 4C), during which the rats were again free to use either paw, the sham control animals did not exhibit any remaining bias in forelimb use (see Fig. 4). The behaviour of lesion and graft groups was similar to that described during the first phase of the experiment, i.e. they were equally impaired in the use of their contralateral paw. An overall analysis confirmed no difference in the use of the preferred paw $\left(F_{3,37}=1,04 ;\right.$ n.s. $)$, whereas lesion and graft groups were impaired in reaching with the contralateral paw. In particular, the apparent small improvement in the use of the contralateral paw by the neonatal grafted rats (see Fig. $4 C$ ) was not statistically significant $\left(F_{3,37}=17.87, \quad P<0.001\right.$; lesioned $=$ adult graft $=$ neonatal graft $>$ control, $P<0.01)$. The performances using each paw during the first and last stage of the experiment give a non-significant group $\times$ stage interaction $\left(F_{3,37}=1.47 ;\right.$ n.s. $)$.

\section{DISCUSSION}

The present study indicates that in spite of the fact that DA neurons implanted in adult and neonatal rats establish, at least at the gross morphological level, different patterns of anatomical incorporation into the host brain, the two types of graft appear to be functionally equivalent. Thus, grafts implanted into neonatal or adult hosts are similar in their ability to compensate amphetamine-induced rotation and to partially prevent the development of apomorphineinduced rotation following unilateral lesion of the mesotelencephalic pathway, whereas neither type of graft was able to ameliorate the rats' deficits in contralateral forelimb use.

\section{Anatomical characteristics of transplants in the} neonatal vs adult brain

The anatomical characteristics of the transplants differed markedly according to whether the suspension was implanted into adult or neonatal rats. Grafts in adult animals were in all cases well-delineated tissue masses that could be clearly distinguished from the host neostriatum. Such reaggregation of ventral mesencephalic DA grafts has been well described in previous accounts of suspension implants into the adult neostriatum. ${ }^{3.10 .23}$ In contrast, grafts of the same cell suspensions into the neonatal neostriatum did not generally hecome established as self-contained tissue masses. Rather, TH-positive neurons were seen scattered throughout the neostriatum, suggesting an interdigitation or migration of the cells within the neonatal environment. This migration could in some cases take place over substantial distances. Only occasionally are small self-contained clumps of graft tissue observed following neonatal implants, and these seem to occur when the grafted cells have become located in a natural space or fissure, such as in the lateral ventricle, in which case they appear to 
reaggregate together. Only in such cases can the survival of non-dopaminergic cells of graft origin be observed. Although there is no reason to suspect that other non-dopaminergic neurons have not survived within the dispersed grafts, this will require further studies using explicit labelling of grafted cells ${ }^{14}$ to ascertain their more general survival after implantation into the neonatal brain. The different morphological characteristics of mesencephalic DA grafts in the neonatal brain are also consistent with previous accounts. ${ }^{44}$ The counts of TH-positive cells implanted in neonatal hosts were approximately half those obtained in the adult hosts, but in view of the degree of variability between animals this difference did not achieve statistical significance.

\section{Anatomical characteristics of transplants in the intact vs denervated brain}

Doucet and colleagues ${ }^{23}$ reported that both the size of the somata of DA neurons and the extent of their fibre outgrowth were significantly greater in grafts implanted in a neostriatum which had been previously deprived of its DA afferents. In agreement with the Doucet study, we have found that the grafts implanted into the intact neostriatum and receiving 6-OHD $\Lambda$ lesions two months later (group adult graft) contained a similar number of neurons to the grafts in animals receiving the lesions three weeks earlier (group lesion then adult graft), confirming that the presence of a lesion is not critical for cell survival. However, whereas Doucet and colleagues ${ }^{23}$ found that the outgrowth into the intact neostriatum (as unmasked by an acute lesion made only days before killing) was relatively sparse, we have found here that similar grafts implanted into the intact neostriatum can give rise to a relatively extensive fibre outgrowth if the grafts are allowed to survive, prior to killing, for a period of several months after the lesion. Indeed, the extent of axonal outgrowth was fully comparable with that observed in animals of the lesion then adult graft group in the present study and in other previous reports of grafts implanted into lesioned neostriatum. $3,0,31,33,44$ Thus, the capacity for extensive fibre outgrowth that is routinely reported to take place when DA grafts are implanted into the denervated neostriatum is not necessarily restricted to immature neurons growing into denervated targets. Even DA neurons in relatively well-established grafts can undergo extensive axonal elongation in response to the stimulus provided by subsequent target denervation, although the present behavioural data suggest that this response from mature neurons may be slower than that observed from embryonic DA cells implanted directly into a denervated environment.

\section{Functional effects of the transplants}

The prophylactic effects of the grafts have been investigated with two kinds of behavioural tasks. Firstly, the grafted rats have been tested in two drug-induced rotation tests which are known to be sensitive for revealing the efficacy of DA transplants in adult or neonatal hosts with nigrostriatal lesions. Secondly, the preventive effect of neonatal grafts on aphagia and adipsia is now shown not to affect another test, skilled paw reaching, which has previously been resistant to amelioration by DA transplants implanted in lesioned adult rats.

Drug-induced turning behaviour. The capacity of intrastriatal DA grafts to compensate the ipsilateral rotation induced by amphetamine in adult rats with unilateral nigrostriatal damage is well established. $8,9,24,27,31,33,38$ Moreover, neonatal DA transplants have also been found to have a similar influence on this drug-induced behaviour after nigrostriatal lesions in newborn rats. ${ }^{44}$ The present study compares the effects of neonatal or adult grafts on the rotation deficit induced by a subsequent nigrostriatal lesion and is noteworthy in several respects.

Firstly, where as the grafts were protective against the development of denervation-related supersensitivity as revealed by the apomorphine rotation, they were not protective against the strong rotation deficits in amphetamine rotation that were apparent immediately following the lesions. This result suggests that different mechanisms are involved in the recovery of these two drug-induced behaviours following transplantation. It is worthy to mention that adrenal and nigral grafts can show different profiles of action on amphetamine- and apomorphine-induced rotation, ${ }^{12}$ confirming that it cannot be a single mechanism. It has been shown that DA grafts implanted within the intact striatum, contralateral to the lesioned side, can decrease apomorphine-induced circling. ${ }^{53}$ These authors hypothesized that the diffusion of dopamine in the absence of reinnervation by DA implanted neurons of the lesioned striatum may be sufficient to compensate the apomorphine-induced rotation. Furthermore, it may be that over the few days following the lesion, DA grafted neurons release enough dopamine over a sufficient area to prevent the development of DA postsynaptic hypersensitivity. but not enough to affect amphetamine-induced rotation.

Secondly, the fact that the grafts had no prophylactic effect on amphetamine-induced rotation contrasts with our previous study, ${ }^{33}$ in which DA grafts implanted into the intact neostriatum of adult rats appeared to have a partially protective effect against nigrostriatal lesions made four months after transplantation. However, several differences in the protocols may explain this discrepancy. (1) In our former experiment the grafted rats had experienced amphetamine four times before the lesion, whereas in the present experiment they had no prelesion tests. This difference may be important since several lines of evidence indicate that the functional efficacy of DA grafts is increased both after pharmacological priming ${ }^{6,2,43}$ and by repeated testing postgrafting. ${ }^{54}$ (2) In the former study we lesioned the DA system four 
months after grafting, which may have allowed relatively slow restorative growth to become established even in the intact neostriatum, beyond that achieved with the shorter two month delay in the present experiment. (3) An apparent prophylactic effect was observed in three of the neonatally grafted animals in the present experiment, and these same three had the greatest number of surviving TH-positive neurons. Although detailed cell counts were not conducted in the previous experiment, a good prophylactic response may be dependent on the survival of a large number of DA neurons than the 200 or so necessary for a good functional response in the lesioned brain. $^{13,40}$

Thirdly, the grafts did develop the capacity to compensate amphetamine rotation but this was slower to develop than is typically observed when embryonic grafts are implanted directly into the denervated neostriatum. Thus, in the present study, most animals grafted prior to lesioning (adult and neonatal) did manifest recovery of the marked amphetamine rotation, indicating that grafted tissue is functional, but this required an interval of three to seven months postlesion to develop, in contrast to the six weeks to two months typically required for full compensation following grafting of DA neurons into the denervated neostriatum. Comparison with the lesion then adult graft group is informative in this respect. The lesion then adult graft rats were part of a different experiment, although they received their graft implants from the same cell suspensions as used in the main experimental neonatal and adult graft groups. Nevertheless, we report here on the functional changes in amphetamine rotation in this group because this measure indicates that the graft tissue itself was viable, and produces a time course of recovery in the standard lesion paradigm that is directly comparable with previous reports. Thus, the slow response in the neonatal and adult graft groups is not simply because this experiment was based on a poor batch of graft tissue. In the light of the observations of Doucet and colleagues, ${ }^{23}$ it is likely that the present grafts, whether implanted into the neonatal or the adult brain, did not undergo extensive initial outgrowth in the intact neostriatum. Rather, the extensive outgrowth that was finally observed most probably became established in response to the stimuli associated with target denervation following the lesion. It is most plausible to suppose that the slow development of the functional response is due to a less vigorous fibre outgrowth from mature neurons in well-established grafts than is typically observed from embryonic neurons freshly implanted into a lesion environment. This issue clearly warrants more detailed study by direct analysis of the time course of fibre outgrowth in response to lesions made at different times before or after the graft implantation.

Fourthly, at no stage of the study did the quality or magnitude of any functional response differ between animals receiving DA grafts as neonates or as adults. This is apparent not just in the time course of the compensation of the amphetamine rotation, but also in the comparable blockade of the apomorphine rotation response in both neonatal and adult graft groups. Thus, although there may have been substantial differences in the gross morphology of the grafts in the neonatal and adult brain, the functional response in this respect is comparable. This suggests that the individual dopaminergic (and other) neurons in the gratts establish similar patterns of connectivity with the host brain whether those cells are organized as discrete tissues or dispersed within the host neostriatum. In contrast to the several studies suggesting reciprocal contacts between grafts implanted in the denervated adult neostriatum, ${ }^{34,48}$ the connectivity of DA grafts implanted either into the neonatal brain or into the intact neostriatum has not yet been evaluated at the ultrastructural level.

Skilled forelimb use. Dopaminergic grafts were not able to alleviate the deficit in skilled paw reaching in recipients of either age group. This absence of effect is not due to poor survival of the graft or their failure to reinnervate the host tissue. This is indicated both by the anatomical findings and the functional response measured by the recovery in apomorphine rotation when skilled paw reaching was tested. Grafted animals were found to compensate amphetamine-induced rotation when tested two days following the completion of the skilled paw reaching task, i.e. seven months after the lesion. In view of the short interval between the two behavioural tasks, this result indicates that, at least for the last part of the paw reaching task, DA grafts were able to influence amphetamine-induced rotation without affecting the deficit in motor skills.

One possible reason for this lack of effect is that the grafts did not reinnervate other terminal fields denervated by the lesion which might be critical in limb use. It has been suggested that combined lesions of the nucleus accumbens and the neostriatum yield greater deficits in skilled paw reaching than DA depletion of the neostriatum alone ${ }^{50}$ Consequently, the failure to restore skilled paw reaching could be due to the lack of simultaneous reinnervation of both the nucleus accumbens and the neostriatum by DA grafts. The fact that neonatally grafted animals, in which THpositive neurons were scattered within the striatal complex, exhibited a similar deficit in skilled paw reaching to adult grafted animals seems to contradict such a hypothesis. Further, we have recently shown that the simultaneous reinnervation of the nucleus accumbens and the neostriatum, by DA neurons transplanted into adult brain, does not restore skilled use of the contralateral limb..$^{5}$ Since other telencephalic structures, not reinnervated by the transplants, might be involved in limb use, ${ }^{67}$ the question of the requirement for a simultaneous restoration of a DA tone within several terminals fields of the DA system for the performance of normal behaviour remains open. 
An alternative explanation for the failure of recovery of skilled paw reaching is that this aspect of motor function is dependent upon a functionally intact nigrostriatal circuitry, which is not restored by nigral grafts implanted into an ectopic striatal location. In particular, the activity of the DA grafted neurons may not be regulated by physiological afferents which normally modulate the DA release from the mesencephalic DA neurons in situ. ${ }^{42}$ Skilled paw reaching and other complex behaviours, which are not restored after transplantation, ${ }^{2,20,21,25,28,30,31,41,43,49}$ may require a dynamic interaction between the activity of the DA neurons and both internal and external stimuli. "In agreement with this hypothesis, striatal grafts which have the capacity to restore more extensive reciprocal connections between the grafts and the host brain ${ }^{22,57}$ are effective in ameliorating paw reaching ability. ${ }^{32,54}$ In a similar vein, Herman and colleagues have shown that deficits in hoarding and exploratory behaviour are compensated by DA neurons implanted in the nucleus accumbens only if the grafts are stimulated by a low dose of amphetamine. The authors hypothesized that "the effect of D-amphetamine pre-treatment would be to mimic the lacking interneuronal stimulations needed for performing the required task". "The failure of the dopaminergic grafts implanted into the neonatal brain to provide any greater protective effect than when implanted into the adult brain suggests that neonatal implantation is ineffective in restoring a fuller degree of circuit reconstruction necessary for recovery of skilled reaching performance, in spite of the better incorporation that had been suggested by the previous studies indicating that such treatments were sufficient to restore regulatory eating and drinking behaviours following subsequent lesions. ${ }^{58.59}$

\section{CONCLUSIONS}

The aim of this experiment was to examine the roles of timing of the lesion (implantation into intact hosts with subsequent lesions vs that into neonatally lesioned hosts, respectively) and of the behavioural tasks (aphagia and adipsia vs drug-induced behaviours, displacement activities, learning behaviours, innate behaviour) on the functional efficacy of neural grafts implanted into neonatal host brain. Dopaminergic transplantation into neonatal hosts provides little advantage over implantation into adults in restoring skilled paw reaching when the grafts are implanted in the intact brain, in agreement with previous reports with rotation and more complex behaviours, ${ }^{2.44}$ and in contrast to the benefit provided by neonatal grafts on the eating and drinking deficits. ${ }^{58,59}$ This suggests that the preventive effect afforded by grafts in newborn rats may be limited to restricted classes of behaviour, such as lesion-induced aphagia and adipsia, and does not provide a general strategy for achieving improved survival, greater anatomical connectivity or more extensive functional recovery than the standard strategy of implantation into adults.

Acknowledgements - This work was supported by an MRC grant (to S.B.D), and by grants from INSERM and INSERM-MRC, the Association "Fondation de France" and the Association "Fondation Simone et Cino Del Duca" (to D.N.A.).

\section{REFERENCES}

1. Abercrombie M. (1946) Estimation of nuclear population from microtome sections. Anat. Rec. 94, 239-249.

2. Abrous D. N., Choulli K., Rouge-Pont F., Simon H., Le Moal M. and Herman J. P. (1992) Effects of intracerebral dopaminergic grafts on behavioural deficits induced by nconatal 6-OHDA lesions of the mesotelencephalic dopaminergic pathway. Neuroscience 54, $499-511$.

3. Abrous N., Guy J., Vigny A., Calas A., Le Moal M. and Herman J. P. (1988) Development of intracerebral dopaminergic grafts: a combined immunohistochemical and autoradiographic study of its time course and environmental influences. J. comp. Neurol. 273, 26 . 41.

4. Abrous D. N., Stinus L., Le Moal M. and Herman J. P. (1990) Locomotor hyper-response to intra-accumbens D-Ala-Met-enkephalin following the lesion of the mesocorticolimbic dopaminergic pathway: reversal by intra-accumbens implants of embryonic dopaminergic neurons. Brain Res. 525, 155-159.

5. Abrous D. N., Shaltot A., Torres E. M. and Dunnett S. B. (1992) Dopamine-rich grafts in the neostriatum and/or the nucleus accumbens: effects on drug-induced behaviours and skilled paw reaching. Neuroscience 53, 187-197.

6. Abrous D. N., Torres E. M., Annett L. E., Reading P. and Dunnett S. B. (1992) Intrastriatal dopaminergic-rich grafts induce a hyperexpression of Fos protein when challenged with amphetamine. Expl Brain Res. 91, 181-190.

7. Annett L. E., Dunnett S. B., Martel F. L., Rogers D. C., Ridley R. M., Baker H. F. and Mardsen C. D. (1990) A functional assessment of embryonic dopaminergic grafts in the marmoset. Prog. Brain Res. 22, 535-542.

8. Björklund A., Dunnett S. B., Stenevi U., Lewis M. E. and Iversen S. D. (1980) Reinnervation of the denervated striatum by substantia nigra transplants: functional consequences as revealed by pharmacological and sensorimotor testing. Brain Res. 199, 307-333.

9. Björklund A., Schmidt R. H. and Stenevi U. (1980) Functional reinnervation of the neostriatum in adult rat by use of intraparenchymal grafting of dissociated cell suspensions form the substantia nigra. Cell Tiss. Res. 212, 39-45.

10. Björklund A., Stenevi U., Schmidt R. H., Dunnett S. B. and Gage F. H. (1983) Intracerebral grafting of neuronal cell suspensions. II. Survival and growth of nigral cell suspensions implanted in different brain sites. Acta physiol scand. $522,9-18$.

11. Björklund A., Lindvall O., Isacson O., Brundin P., Wictorin K., Strecker R. E., Clarke D. J. and Dunnett S. B. (1987) Mechanisms of action of intracerebral neural implants: studies on nigral and striatal grafts to the lesioned striatum. Trends Neurosci. 10, 509-516. 
12. Brown V. J. and Dunnett S. B. (1989) Comparison of adrenal and foetal nigral grafts on drug-induced rotation in rats with 6-OHDA lesions. Expl Brain Res. 78, 214-218.

13. Brundin P., Isacson O. and Björklund A. (1985) Monitoring of cell viability in suspensions of embryonic CNS tissue and its use as a criterion or intracerebral graft survival. Brain Res. 331, 251-259.

14. Cadusseau J. and Peschanski M. (1992) Identifying grafted cells. In Neural Transplantation: A Practical Approach (eds Dunnett S. B. and Björklund A.), pp. 177-201. IRL Press, Oxford.

15. Castro A. J., Tonder N., Sunde N. Aa. And Zimmer J. (1987) Fetal cortical transplants in the cerebral hemisphere of newborn rats: a retrograde fuorescent analysis of connections. Expl Brain Res. 66, 533-542.

16. Castro A. J., Tonder N., Sunde N. Aa. and Zimmer J. (1988) Fetal neocortical transplants grafted to the cerebral cortex of newborn rats receive afferents from the basal forebrain, locus coeruleus and midline raphe. Expl Brain Res. 69,613-622.

17. Castro A. J., Zimmer J., Sunde N. Aa. and Bold E. L. (1985) Transplantation of fetal cortex to the brain of newborn rats: a retrograde fluorescent analysis of callosale and thalamic projections from the transplant to hose. Neurosci. Lett. 60, 283-288.

18. Change F.-L. F., Steedman J. G. and Lund R. D. (1984) Embryonic cerebral cortex placed in the occipital region of newborn rats makes connections with the host brain. Devl Brain Res. 13, 164-166.

19. Change F.-L. F., Steedman J. G. and Lund R. D. (1986) The lamination and connectivity of embryonic cerebral cortex transplanted into newborn rat cortex. $J$. comp. Neurol. 244, 401-411

20. Choulli K., Herman J. P., Abrous N. and Le Moal M. (1987) Behavioral effects of intra-accumbens transplants in rats with lesions of the mesocorticolimbic dopamine system. Ann. N.Y. Acad. Sci. 495, 497-509.

21. Choulli K., Herman J. P., Rivet J. M., Simon H. and Le Moal M. (1987) Spontaneous and graft-induced behavioural recovery after 6-hydroxydopamine lesion of the nucleus accumbens in the rat. Brain Res, 407, 376-380.

22. Clarke D. J., Dunnett S. B., Isacson O., Sirinathsinghji D. J. S. and Björklund A. (1988) Striatal grafts in rats with unilateral neostriatal lesions-1. Ultrastructural evidence of afferent synaptic inputs from the host nigrostriatal pathway. Neuroscience 24, 791-801.

23. Doucet G., Brundin P., Descarries L. and Björklund A. (1989) Effect of prior dopamine denervation on survival and fiber outgrowth from intrastriatal fetal mesencephalic grafts. Eur. J. Neurosci. 2, 279-290.

24. Dunnett S. B., Björklund A., Stenevi U, and Iversen S. D. (1981) Behavioural recovery following transplantation of substantia nigra in rats subjected to 6-OHDA lesions of the nigrostriatal pathway. I. Unilateral lesions. Rrain Res. 215 , $147-161$.

25. Dunnett S. B., Björklund A., Stenevi U. and lversen S. D. (1981) Behavioural recovery following transplantation of substantia nigra in rats subjected to 6-OHDA lesions of the nigrostriatal pathway. II. Bilateral lesions. Brain Res. 229, $457-470$.

26. Dunnett S. B., Björklund A., Stenevi U. and Iversen S. D. (1981) Grafts of substantia nigra reinnervating the ventrolateral striatum ameliorate sensorimotor impairments and akinesia in rats with 6-OHDA lesions of the nigrostriatal pathway. Brain Res. 229, 209-217.

27. Dunnett S. B., Björklund A., Schmidt R. H., Stenevi U. and Iversen S. D. (1983) Intracerebral grafting of neuronal cell suspensions. IV. Behavioural recovery in rats with unilateral 6-OHDA lesions following implantation of nigral cell suspensions in different forehrain sites. Acta physiol. scand. Suppl. 522, 29-37.

28. Dunnett S. B., Björklund A., Schmidt R. H., Stenevi U. and Iversen S. D. (1983) Intracerebral grafting of neuronal cell suspensions. $\mathrm{V}$. Behavioural recovery in rats with bilateral 6-OHDA lesions following implantation of nigral cell suspensions. Acta physiol. scand. Suppl. 522, 39-47.

29. Dunnett S. B., Bunch S. T., Gage F. H. and Björklund A. (1984) Dopamine-rich transplants in rats with 6-OHDA lesions of the ventral tegmental area. 1. Effects on spontaneous and drug-induced locomotor activity. Behav. Brain Res. $13,71-82$

30. Dunnett S. B., Whishaw I. Q., Jones G. H. and Isacson O. (1986) Effects of dopamine-rich grafts on conditioned rotations in rats with unilateral 6-hydroxydopamine lesions. Neurosci. Lett. 68, 127-133.

31. Dunnett S. B., Whishaw I. Q., Rogers D. C. and Jones G. H. (1987) Dopamine-rich grafts ameliorate whole body motor asymmetry and sensory neglect but not independent limb use in rats with 6-hydroxydopamine lesions. Brain Res. 415, 63-78.

32. Dunnett S. B., Isacson O., Sirinathsinghji D. J. S., Clarke D. J. and Björklund A. (1988) Striatal grafts in rats with unilateral neostriatal lesions--III. Recovery from dopamine-dependent motor asymmetry and deficits in skilled paw reaching. Neuroscience 24, 813-820.

33. Dunnett S. B., Hernandez T. D., Summerfield A., Jones G. H. and Arbuthnott G. (1988) Graft-derived recovery from 6.OHDA lesions: specificity of ventral mesencephalic graft tissues. Expl Brain Res. 71, 411-424.

34. Freund T. F., Bolam J. P., Björklund A., Stenevi U., Dunnett S. B., Powel J. F. and Smith A. D. (1985) Efferent synaptic connections of grafted dopaminergic neurons reinnervating the host neostriatum: a tyrosine hydroxylase immunocytochemical study. $J$. Neurosci. 5, 603-616.

35. Harvey A. R, and Lund R. D. (1981) Transplantation of tectal tissue in rats. II. Distribution of host neurons which project transplants. J. comp. Neurol. 202, 505 -520 .

36. Harvey A. R. and Lund R. D. (1984) Transplantation of tectal tissue in rats. IV. Maturation of transplants and development of host retinal projection. Devl Brain Res. 12, 27-37.

37. Harvey A. R., Golden G. T. and Lund R. D. (1982) Transplantation of tectal tissue in rats III. Functional innervation of transplants by host afferents. Expl Brain Res. 47, 437-445.

38. Herman J. P., Choulli K. and Le Moal M. (1985) Hyper-reactivity to amphetamine in rats with dopaminergic grafts. Expl Brain. Res. 60, 521-526.

39. Herman J. P., Nadaud D., Choulli K., Taghzouti K., Simon H. and Le Moal M. (1985) Pharmacological and behavioral analysis of dopaminergic grafts placed into the nucleus accumbens. In Neural Grafting in the Mammalian CNS (eds Björklund A. and Stenevi U.), pp. 519 527. Elsevicr, Amsterdam.

40. Herman J. P., Choull K., Geffard M., Nadaud D., Taghzouti K. and Le Moal M. (1986) Reinnervation of the nucleus accumbens and frontal cortex of the rat by dopaminergic grafts and effects on hoarding behavior. Brain Res. 372, 210-216.

41. Herman J. P., Choulli K., Abrous N., Dulluc J. and Le Moal M. (1988) Effects of intra-accumbens grafts on behavioral deficits induced by 6-OHDA lesions of the nucleus accumbens or A10 dopaminergic neurons: a comparison. Behav. Brain Res. 29, 73-83. 
42. Herman J. P., Rivet J. M., Abrous N and Le Moal M. (1988) Intracerebral dopaminergic transplants are not activated by electrical footshock stress activating in situ mesocorticolimbic neurons. Neurosci. Lett. 90, 83-88.

43. Herman J. P., Choulli K., Abrous N. and Le Moal M. (1989) Intracerebral grafts of dopaminergic neurons: a discussion of their functional effects and mechanisms of action. In Neuronal Grafting and Alzheimer's Disease (eds Gage $F$. Privat A. and Christen Y.), pp. 21-23. Springer-Verlag, Berlin.

44. Herman J. P., Abrous D. N. and Le Moal M. (1991) Neonatal implantation of embryonic dopaminergic neurons following the unilateral lesion of the nigrostriatal dopaminergic pathway: anatomical characterization and influence on drug-induced rotation. Neuroscience $40,465-476$.

45. Jaeger C. B. and Lund R. D. (1979) Efferent fibers from transplanted cerebral cortex of rats. Brain Res. 165, 338-342.

46. Jaeger C. G. and Lund R. D. (1980) Transplantation of embryonic occipital cortex to the tectal region of newborn rats: a light microscopic study of organization and connectivity of the transplants. $J$. comp. Neurol. 194, $571-597$.

47. Lund R. D. and Harvey A. R. (1981) Transplantation of tectal tissue in rats. I. Organization of transplants and pattern of distribution of host afferents within them. J. comp. Neurol. 201, 191-209.

48. Mahalik T. J., Finger T. E., Strömberg I. and Olson L. (1985) Substantia nigra transplants into the denervated striatum of the rat: ultrastructure of graft and host interconnections. J. comp. Neurol, 240, 60-70.

49. Mandel R. J., Brundin P. and Björklund A. (1990) The importance of graft placement and task complexity for transplant-induced recovery of simple and complex sensorimotor deficits in dopamine denervated rats. Eur. $J$. Neurose $i$. 2, 888-894.

50. Mandel R. J., Brundin P., Wictorin K. and Björklund A. (1991) Evaluation of functional mechanism of fetal mesencephalic grafts placed in the dopamine denervated striatum. Third IBRO World Congress of Neurascience, 49 August, Montreal, Canada, p. 109. Pergamon Press, Oxford.

51. Mayer E., Brown V. J., Dunnett S. B. and Robbins T. W. (1992) Striatal graft-associated recovery of a lesion-induced performance deficit in the rat requires learning to use the transplant. Eur. J. Neurosci. 4, 119-126.

52. McLoon L. K., McLoon S. C., Chang, F.-L. F., Steedman J. G. and Lund R. D. (1985) Visual system transplanted to the brain of rats. In Neural Grafting in the Mammalian CNS (eds Björklund A. and Stenevi U.), pp. 267283. Elsevier, Amsterdam.

53. Mendoza-Ramirez J.-L., Aguilar-Roblero R., Zainos-Rosales A. and Drucker-Colin R. (1991) Fetal substantia nigra and adrenal medullary grafts placed contralateral to the nigrostriatal lesion side induce a decrease in turning behaviour nut not in dopamine receptor density. Rest. Neurol. Neurosci. 3, 177-185.

54. Montoya C. P., Astell S. and Dunnett S. B. (1990) Effect of nigral and striatal grafts on skilled forelimb use in the rat. Prog. Brain Res. 82, 459.466

55. Montoya C. P., Campbell-Hope L. J., Pemberton K. D. and Dunnett S. B. (1991) The "staircase test"; a measure of independent forelimb reaching and grasping abilities in rats. J. Neurosci. Meth. 36, 219-228.

56. Nadaud D., Herman J. P., Simon H. and Le Moal M. (1984) Functional recovery following transplantation of the ventral tegmental mesencephalic cells in rats subjected to 6-OHDA lesions of the mesolimbic dopaminergic neurons. Brain Res. 304, 137-141.

57. Pritzel M., Isacson O., Brundin P., Wiklund L. and Björklund A. (1986) Afferent and efferent connections of striatal grafts implanted into the ibotenic acid lesioned neostriatum. Expl Brain Res. 65, 112-126.

58. Rogers D. C. and Dunnett S. B. (1989) Neonatal dopamine-rich grafts and 6-OHDA lesions independently provide partial protection from the adult nigrostriatal lesion syndrome. Behav. Brain Res. 34, 131-146.

59. Rogers D. C., Martel F. L. and Dunnett S. B. (1990) Nigral cells in neonatal rats protect from aphagia induced by subsequent adult 6-OHDA lesions: the importance of striatal location. Expl Brain Res. 80, 172-176.

60. Schwartz S. S. and Freed W. R. (1987) Brain tissue transplantation in neonatal rats prevents a lesion-induced syndrome of adipsia, aphagia and akinesia. Expl Brain Res. 65, $449-454$.

61. Siegfried B. and Bures J. (1980) Handedness in rats: blockade of reaching behavior by unilateral 6-OHDA injections into substantia nigra and caudate nucleus. Physiol. Psychol. 8, 360-368.

62. Snyder-Keller A. M. and Lund R. M. (1990) Amphetamine sensitisation of stress-induced turning in animals given unilateral dopamine transplants in infancy. Brain Res. 514, 143-146.

63. Snyder-Keller A. M., Carder R. K. and Lund R. M. (1989) Development of dopamine innervation and turning behavior in dopamine-depleted infant rats receiving unilateral nigral transplants. Neuroscience 30, 779-794.

64. Sunde N. Aa., Laurberg S. and Zimmer J. (1984) Brain grafts can restore irradiation-damaged neuronal connections in newborn rats. Nature $310,51-53$.

65. Sunde N. Aa. and Zimmer J. (1983) Cellular, histochemical and connective organization of the hippocampus and fascia dentata transplanted to different regions of immature and adult rat brain. Devl Brain Res. 8, 165-191

66. Ungerstedt U. and Arbuthnott G. W. (1970) Quantitative recording of rotational behaviour in rats with 6-hydroxydopamine lesions of the nigrostriatal system. Brain Res. 24, 485-493.

67. Whishaw I. Q., Castañeda E. and Gorny B. P. (1992) Dopamine and skilled limb use in the rat: more severe bilateral impairments follow substantia nigra then sensorimotor cortex 6-hydroxydopamine injection. Behav. Brain Res. 47, 89-92.

68. Whishaw I. Q., O'Connor W. T. and Dunnett S. B. (1986) The contributions of motor cortex, nigrostriatal dopamine and caudate putamen to skilled forelimb use in the rat. Brain 109, 805-843.

69. Zimmer J. and Sunde N. Aa. (1984) Neuropeptides and astroglia in intracerebral hippocampal transplants: an immunohistochemical study in the rat. J. comp. Neurol. 227, 331-347. 